KIAS-P04050

UOSTP-04105

hep-th/0412170

\title{
Separation of Spontaneous Chiral Symmetry Breaking and Confinement via AdS/CFT Correspondence
}

\author{
Dongsu Bak ${ }^{a}$ and Ho-Ung Yee ${ }^{b}$ \\ ${ }^{a}$ Physics Department, University of Seoul, Seoul 130-743, Korea, \\ ${ }^{b}$ School of Physics, Korea Institute for Advanced Study \\ 207-43, Cheongryangri-Dong, Dongdaemun-Gu, Seoul 130-722, Korea \\ (dsbak@mach.uos.ac.kr, ho-ung.yee@kias.re.kr)
}

\begin{abstract}
We analyze, in the framework of AdS/CFT correspondence, the gauge theory phase structure that are supposed to be dual to the recently found non-supersymmetric dilatonic deformations to $A d S_{5} \times S^{5}$ in type IIB string theory. Analyzing the probe D7-brane dynamics in the backgrounds of our interest, which corresponds to the fundamental $\mathcal{N}=2$ hypermultiplet, we show that the chiral bi-fermion condensation responsible for spontaneous chiral symmetry breaking is not logically related to the phenomenon of confinement.
\end{abstract}




\section{Introduction}

Quantum Chromodynamics (QCD) is believed to describe hadrons in the universe. While much of its perturbative dynamics is by now fairly well understood, it is still hard to analyze, in convincing ways, many non-perturbative phenomena that are relevant in low energy regime. Among these are the confinement of quarks and the spontaneous breaking of their (approximate) chiral symmetry $(\mathrm{S} \chi \mathrm{SB})$. Although these two aspects of QCD have their same origin in strongly interacting dynamics, there hasn't been found no logical connection between the two phenomena. In this paper, we provide, in our belief, one convincing example showing the logical separation between confinement and S $\chi \mathrm{SB}$. Our analysis seems to suggest that spontaneous chiral symmetry breaking of massless quarks may happen without any need of a confining potential between them.

Our analysis is based on the proposal of AdS/CFT correspondence, in which Type IIB string theory on $A d S_{5} \times S^{5}$ background is equivalent to the $\mathcal{N}=4 \mathrm{SYM}$ theory on the boundary of $A d S_{5}$ [1]. The duality between the two descriptions is supposed to hold even at the level of Hilbert spaces of their corresponding quantum theory; (semi-classical) deformations of $A d S_{5} \times S^{5}$ which vanish asymptotically at the boundary correspond to some quantum states in the dual gauge theory [2, 3]. Depending on the deformations in the bulk that we are considering, these states share several interesting properties with the usual vacuum states of realistic gauge theories, such as homogeneity over space and non-vanishing gluon condensation, etc. Henceforth, studying confinement and $\mathrm{S} \chi \mathrm{SB}$ on these states may give us an important laboratory for unraveling the relation between the two phenomena.

The deformed backgrounds of our interest are a family of dilatonic deformations of $A d S_{5} \times S^{5}$ that were found in Ref. 4]. A nice fact about these solutions is the existence of a single adjustable parameter, $k / \mu$, which enables us to scan a range of corresponding quantum states. In the gauge theory side, this parameter represents the ratio of the gluon condensation to the energy density of the quantum states. The analysis in Ref. 4] showed that for $k / \mu<-12$, the potential between (heavy) quark/anti-quark pair is confining, while states of $k / \mu>-12$ were argued to exhibit Coulomb-like behavior. However, in section 3, we perform a more careful study for the cases of $k / \mu>-12$ to find the screening phase instead for them. We also look at the response to magnetically charged objects and get an interesting phase structure.

To study $\mathrm{S} \chi \mathrm{SB}$ on these background states, a small number of light quarks/anti-quarks are introduced a la Karch and Katz in section 4; probe D7-branes [] $]$. They are $N_{f}, \mathcal{N}=2$ hypermultiplets in fundamental representation of the $S U(N)$ gauge group, and their effect to $\mathcal{N}=4 S U(N)$ SYM dynamics may be neglected in $N \gg N_{f}$ limit via quenched approximation. 
D7-probe for studying $\mathrm{S} \chi \mathrm{SB}$ was analyzed first in [8], and its use also for hadron physics [6, 7, 9, 10, 11] is by now a well established method in the literature ( See [12, 13, 14, 15, 16, for other set-up's of introducing flavors.). From a careful numerical work, we seem to find a convincing evidence that $\mathrm{S} \chi \mathrm{SB}$ persists in the region of our parameter space in which the confinement no longer exists. Therefore, on the basis of validity of the AdS/CFT correspondence, it is clear that some states in large $N \mathcal{N}=4$ SYM theory, which have non-vanishing gluon condensation, serve as a rare ground for logical separation between $\mathrm{S} \chi \mathrm{SB}$ and confinement. We summarize and conclude in section 5 .

\section{Bulk Solutions: Dilatonic Deformation in $A d S_{5} \times S^{5}$}

In Ref. 4], a family of non-supersymmetric solutions of type IIB supergravity with asymptotic $A d S_{5} \times S^{5}$ geometry were found by turning on generic dilaton deformation to the maximally supersymmetric $A d S_{5} \times S^{5}$ background $^{1}$ (For an earlier example, see [17, 18]). Analytic solutions are available only for the cases in the Poincare patch, which preserves $\mathbf{R} \times I S O(3) \times S O(6)$ subgroup of the full $S O(2,4) \times S O(6)$ symmetry of $A d S_{5} \times S^{5}$. Explicitly, these solutions are

$$
\begin{aligned}
d s^{2} & =(y-b)^{\frac{1-a}{4}}(y+b)^{\frac{1+a}{4}}\left(-\left(\frac{y-b}{y+b}\right)^{a} d t^{2}+\frac{d y^{2}}{16(y-b)^{\frac{5-a}{4}}(y+b)^{\frac{5+a}{4}}}+d \vec{x}^{2}\right)+d \Omega_{5}^{2} \\
\phi & =\phi_{0}+\frac{k}{8 b} \log \left(\frac{y-b}{y+b}\right), \quad F_{5}=Q\left(\omega_{5}+* \omega_{5}\right)
\end{aligned}
$$

where the metric is in the Einstein frame and we let the AdS radius be unity for simplicity. Here $Q$ is the constant that counts the number $N$ of D3-branes, $d \Omega_{5}^{2}$ and $\omega_{5}$ are the metric and the volume form of unit five sphere, respectively. Clearly, the $S^{5}$ part of the original $A d S_{5} \times S^{5}$ is intact and the $S O(6) \mathrm{R}$-symmetry of the $\mathcal{N}=4 \mathrm{SYM}$ theory is unbroken at this level. The parameters $a$ and $b$ are defined in terms of two quantities, $k$ and $\mu$;

$$
a \equiv\left(1+\frac{k^{2}}{6 \mu^{2}}\right)^{-\frac{1}{2}} \quad, \quad b \equiv \frac{\mu}{2}\left(1+\frac{k^{2}}{6 \mu^{2}}\right)^{\frac{1}{2}} .
$$

The solutions have a time-like naked singularity at $y=b$. Up to over-all scaling, these solutions are parameterized by essentially a single variable $k / \mu$. They can be thought of as describing some quantum states in the bulk $A d S_{5}$ spacetime, because their deformations to the maximally

\footnotetext{
${ }^{1}$ It is also possible to have solutions with the axion field turned on, but these solutions are readily obtained from the current ones by $S L(2, Z)$ action. See also Ref. [19] for the nonsingular class of dilatonic deformation in $A d S_{5}$.
} 
supersymmetric $A d S_{5} \times S^{5}$ solution decay sufficiently fast as we approach the boundary. According to AdS/CFT correspondence, we therefore interpret them as the dual geometries of some quantum states of the $\mathcal{N}=4$ SYM gauge theory living on the boundary $R^{1,3}$.

An element of the standard AdS/CFT dictionary gives us an important information about these quantum states in the gauge theory. In terms of the coordinate $r$ defined by $r^{2}=\sqrt{\frac{b}{2}} e^{s}$ and $y=b \cosh (2 s)$, the bulk metric goes to the standard $A d S_{5} \times S^{5}$ metric for large $r$, and $r$ becomes the usual radial coordinate of asymptotic $A d S_{5}$. The dilaton field then asymptotes to

$$
\phi=\phi_{0}+\frac{k}{8 b} \log \left(\frac{y-b}{y+b}\right) \sim \phi_{0}-\frac{k}{4} \frac{1}{r^{4}},
$$

which implies that the corresponding quantum states in the gauge theory have a non-vanishing expectation value of $\mathcal{L}_{\mathrm{CFT}} \sim \frac{1}{2 g_{Y M}^{2}} \operatorname{tr} F^{2}$;

$$
\left\langle\mathcal{L}_{\mathrm{CFT}}\right\rangle=\frac{k}{4}
$$

The ADM energy density of these states was calculated to be proportional to $\mu$.

\section{Phases of Dual $\mathcal{N}=4$ SYM States : Confinement vs Screening}

The family of supergravity backgrounds in the previous section with varying dilaton profile are supposed to describe some quantum states of $\mathcal{N}=4$ SYM theory on $R^{1,3}$. According to AdS/CFT dictionary, these states are characterized by expectation values of $\operatorname{tr} F^{2}$ and the Hamiltonian density. Roughly, we have seen that

$$
\begin{aligned}
& k \sim \frac{1}{2 g_{Y M}^{2}}\left\langle\operatorname{tr}\left(F^{2}\right)\right\rangle=\frac{1}{2 g_{Y M}^{2}}\left\langle\operatorname{tr}\left(\vec{E}^{2}-\vec{B}^{2}\right)\right\rangle, \\
& \mu \sim \frac{1}{2 g_{Y M}^{2}}\left\langle\operatorname{tr}\left(\vec{E}^{2}+\vec{B}^{2}\right)\right\rangle=\mathcal{E},
\end{aligned}
$$

where we denote the energy density by $\mathcal{E}$. Though these states are quantum states of the superconformal $\mathcal{N}=4 \mathrm{SYM}$ theory, they have certain properties that mimic those of interesting vacuum states of more realistic gauge theories; they are homogeneous over spatial $R^{3}$ and have non-vanishing gluon condensation. The latter property has long been suspected of being one of the crucial characteristics of QCD vacuum [20]. It is thus a meaningful endeavor to study quantum structure of these states and talk about their "phases". One has to bear in mind that the strength of the gluon condensate here characterizes the macroscopic states of the $\mathcal{N}=4$ SYM theory and works as a tunable parameter.

One of the key aspects of a given phase of gauge theory is how it reacts to external charges. In the screening phase, external charges are compensated by conducting currents, and subsequently 
screened within some characteristic length scale. Equivalently, the gauge boson gets massive and does not propagate beyond its mass scale. On the other hand, confining phase does not break gauge symmetry and charge conservation. Instead, electric flux is confined to a narrow string, resulting in a linear potential between two charges. One of the most profound observations in gauge theory is that magnetic screening due to condensation of magnetically charged object leads to electric confinement and vice versa. However, there is a caveat here, that is, if there is also a condensation of electrically charged object at the same time, electric confinement will be ruined. In this case, the most plausible expectation is that both electric and magnetic charges are screened.

In this section, we analyze the response of our states of $\mathcal{N}=4 \mathrm{SYM}$ theory to various types of external charges, and find some aspects of interesting phases that we discussed in the above. In the spirit of AdS/CFT correspondence, external charges are described by stretched strings in the supergravity background to the boundary [21,22]. The Wilson line expectation value is obtained from the effective world-sheet dynamics of the stretched strings in the supergravity background. For electric charges, the world-sheet dynamics is dictated by F1 Nambu-Goto action, while magnetic or dyonic cases will be described by D1-DBI action with/without world-sheet gauge flux turned on.

\subsection{Electric Confinement/Screening Transition}

Electric confinement in the above dilatonic backgrounds has been shown to occur in Ref. [4 for $k / \mu<-12$. A Nambu-Goto string stretched between heavy quark/anti-quark pair through the bulk corresponds to the Wilson loop in the dual gauge theory side 21, 22]. In the large AdS radius limit, the string behaves classically and one may get the interaction potential via classical analysis of the Nambu-Goto string dynamics. Here we would like to analyze more general cases including magnetic charges as well.

To deal with general $(p, q)$ string, let us begin with the Dirac-Born-Infeld action,

$$
S=-\frac{1}{2 \pi \alpha^{\prime}} \int d \tau d \sigma e^{-\phi} \sqrt{-\operatorname{det}\left(g_{\mu \nu} \partial_{a} X^{\mu} \partial_{b} X^{\nu}+2 \pi \alpha^{\prime} F_{a b}\right)},
$$

where $g_{\mu \nu}$ is the string frame metric which is related to the Einstein frame metric by

$$
g_{\mu \nu}=e^{\frac{\phi}{2}} g_{\mu \nu}^{E} .
$$

Denoting

$$
M=-\operatorname{det}\left(g_{\mu \nu} \partial_{a} X^{\mu} \partial_{b} X^{\nu}\right),
$$


the Lagrangian density may be written as

$$
\mathcal{L}=-\frac{1}{2 \pi \alpha^{\prime} e^{\phi}} \sqrt{M-\left(2 \pi \alpha^{\prime} E\right)^{2}},
$$

where $E=F_{01}$. Let us introduce the displacement $D$ by

$$
D=\frac{\partial \mathcal{L}}{\partial E}=\frac{2 \pi \alpha^{\prime} E}{e^{\phi} \sqrt{M-\left(2 \pi \alpha^{\prime} E\right)^{2}}} .
$$

$D$ is conserved and $\partial_{\sigma} D=0$; one may obtain an equivalent description of the system by the Legendre transformation,

$$
\mathcal{L}^{\prime}=-D \cdot E+\mathcal{L}=-\frac{1}{2 \pi \alpha^{\prime} e^{\phi}} \sqrt{M\left(1+e^{2 \phi} D^{2}\right)}
$$

by eliminating $E$ using (3.10). The displacement $D$ counts the number of fundamental strings immersed and it is quantized to take an integer value, which we denote as $p$. Using the Einstein frame metric, the above Lagrangian density may be written as

$$
\mathcal{L}=-\frac{1}{2 \pi \alpha^{\prime}} \int d \sigma \sqrt{-\operatorname{det}\left(g_{\mu \nu}^{E} \partial_{a} X^{\mu} \partial_{b} X^{\nu}\right)} \sqrt{p^{2} e^{\phi}+q^{2} e^{-\phi}}
$$

where we also introduced an integer q counting the number of D-strings. The derivation of the $(p, q)$ string action here is only for $q=1$, but we generalize it for an arbitrary $q$. From the above action for the $(p, q)$ string, the S-duality of the IIB string theory is manifest. Namely, the above is invariant under the transformation,

$$
g_{E \mu \nu}^{\prime}=g_{E \mu \nu}, \quad \phi^{\prime}=-\phi, \quad p \leftrightarrow q
$$

Note that in our dilaton-deformed solutions, the S-duality corresponds to simply changing $k \rightarrow$ $-k$ and $\phi_{0} \rightarrow-\phi_{0}$. ¿From this S-duality transformation, it is clear that magnetic charges are confined for $k / \mu>12$, as electrically charged quarks are confined if $k / \mu<-12$.

To see the details of the interaction and the phase structure, let us assume that the $(p, q)$ string is static and choose the gauge $\tau=t$ and $\sigma=y$. We shall consider the case where the $(p, q)$ string trajectory is independent of $x_{2}$ and $x_{3}$. The $(p, q)$ string Lagrangian then becomes

$$
\mathcal{L}=-\sqrt{\lambda} \int d y \sqrt{A(y)\left(B(y)+C(y)(d x / d y)^{2}\right)},
$$

where $\lambda=g_{Y M}^{2} N$ is the t'Hooft coupling and

$$
\begin{aligned}
& A(y)=(y-b)^{\frac{1+3 a}{4}}(y+b)^{\frac{1-3 a}{4}}\left(p^{2} e^{\phi_{0}}\left(\frac{y-b}{y+b}\right)^{\frac{k}{8 b}}+q^{2} e^{-\phi_{0}}\left(\frac{y-b}{y+b}\right)^{-\frac{k}{8 b}}\right), \\
& B(y)=\frac{1}{16(y-b)(y+b)}, \\
& C(y)=(y-b)^{\frac{1-a}{4}}(y+b)^{\frac{1+a}{4}} .
\end{aligned}
$$


The computation showing heavy quark confinement follows closely the one in Ref. [4. The equation of motion,

$$
\frac{d}{d y}\left(\frac{\sqrt{A} C d x / d y}{\sqrt{B+C(d x / d y)^{2}}}\right)=0,
$$

may be integrated once, and one gets

$$
\frac{\sqrt{A} C d x / d y}{\sqrt{B+C(d x / d y)^{2}}}= \pm q^{-2}
$$

with an integration constant $q^{2}$. To understand the dynamical implication, we rewrite (3.17) into the form

$$
\left(\frac{d y}{d x}\right)^{2}+V(y)=0
$$

with the potential

$$
V(y)=\frac{C}{B}\left(1-q^{4} A C\right) .
$$

This can be viewed as a particle moving in one dimension under the potential $V$, regarding the coordinate $x$ as the 'time'.

The confinement occurs when the 'particle' spends an arbitrarily large 'time' when it approaches the turning point denoted by $y_{0}$. At the turning point, one has $d y / d x=0$ and thus $V\left(y_{0}\right)=0$, which implies that

$$
q_{0}^{4} A\left(y_{0}\right) C\left(y_{0}\right)=1,
$$

for an appropriate choice of the integration constant $q=q_{0}$. The condition of spending arbitrarily large 'time' is fulfilled if $V^{\prime}\left(y_{0}\right)=0$. This leads to

$$
\left.(A C)^{\prime}\right|_{y=y_{0}}=0
$$

where the condition $V\left(y_{0}\right)=0$ is used.

Let us first consider the case of $(1,0)$ string connecting electrically charged quark/anti-quark pair. In this case, the latter condition is solved by

$$
y_{0}=-a b-k / 4
$$

For the existence of the solution in the range $y \in(b, \infty)$, one has to impose

$$
y_{0}-b=-a b-k / 4-b \equiv 2 b \beta>0
$$

which is equivalent to

$$
\frac{k}{\mu}<-12
$$


Then $V\left(y_{0}\right)=0$ is satisfied by choosing the integration constant $q$ as

$$
q_{0}^{4}=\frac{1}{2 b} \beta^{\beta}(1+\beta)^{-(1+\beta)} e^{-\phi_{0}}
$$

For small $q$, the separation between the quark/anti-quark pair is of the order of $q$ according to the IR/UV relation. The energy scale here is much higher than that of the confinement. Thus the quark/anti-quark potential for sufficiently small separation is of Coulomb type as expected.

When $\beta>0$ and $q$ approaches $q_{0}$ from below, the string spends more and more 'time' near the turning point $y \sim y_{0}$. The separation between the quark and anti-quark becomes larger and larger as one sends $q$ to $q_{0}$ from below, because the 'time' spent near the turning point increases more and more.

In the limit $q \rightarrow q_{0}$, we can compute the tension of the string and the energy scale of confinement. The energy of the string is given by

$$
E_{s}=\sqrt{\lambda} \int d y \sqrt{A\left(B+C(d x / d y)^{2}\right)}=\sqrt{\lambda} \int d x \sqrt{q^{4} A^{2} C^{2}}
$$

where we have used the equation of motion. The integral in fact diverges and one may regulate it by subtracting the self-energy of quark and anti-quark.

Since $q_{0}^{4} A\left(y_{0}\right) C\left(y_{0}\right)=1$ and the string stays near the turning point for most of the 'time', we find from (3.26) the tension of the confining string to be

$$
T_{Q C D}=\sqrt{\lambda} \sqrt{A\left(y_{0}\right) C\left(y_{0}\right)}=\sqrt{\lambda} q_{0}^{-2}=\sqrt{\lambda \mu} \frac{(1+\beta)^{\frac{1+\beta}{2}}}{\sqrt{a} \beta^{\frac{\beta}{2}}} e^{\frac{\phi_{0}}{2}} .
$$

This sets the scale of confinement. Our result agrees with the previously calculated one in the $\mu \rightarrow 0$ limit [23].

\subsection{Screening}

In the analysis of Ref. 4, the region of $k / \mu>-12$ corresponding to $\beta<0$ was not carefully analyzed because the paper mainly concerned only about the existence of confinement phenomena. We would like to show that this region corresponds, in fact, to the screening phase. In this region the potential $V$ always has a turning point beyond which the singularity is located. This feature of inaccessibility to the singularity is true for all value of the integration constant $q^{2}$. One may ask the following. May an infinitely large separated quark/anti-quark pair be connected through this string solution, by adjusting the integration constant $q$ ? The answer turns out to be no. Namely, there is an upper limit on the separation length between the quark and anti-quark pair in the above solutions of string configuration. 
To show this, let us first note that the separation length is given by

$$
L=2 \int_{y_{\star}}^{\infty} d y \frac{1}{\sqrt{-V}}=2 \int_{y_{\star}}^{\infty} d y \frac{\sqrt{B}}{\sqrt{C} \sqrt{q^{4} A C-1}}
$$

where $y_{\star}$ is the turning point. For small $q$ satisfying the condition $e^{\phi_{0}} b q^{4} \ll 1$, the turning point $y_{\star} \sim 1 /\left(e^{\phi_{0}} q^{4}\right)$ is much larger than $b$ and, thus, the potential $V$ may well be approximated by

$$
V \sim 16 y^{\frac{5}{2}}\left(1-e^{\phi_{0}} q^{4} y\right)
$$

Then the separation is approximately given by

$$
L \sim \frac{e^{\frac{\phi_{0}}{4}} q}{2} \int_{1}^{\infty} \frac{d t}{t^{\frac{5}{4}}} \frac{1}{\sqrt{t-1}} .
$$

This is the case of sufficiently small separation and the expression for the separation is essentially same as the one for the strings in the pure AdS case, because the strings are staying in the near boundary region of the asymptotically AdS space.

To study the upper limit on the separation distance, one should look at the large $q$ behavior of $L$. When $e^{\phi_{0}} b q^{4} \gg 1$, the contribution of the integral from infinity to $y-b=O(b)$ is of order $1 /\left(e^{\frac{\phi_{0}}{2}} q^{2}\right)$, which is small. The turning point occurs in the regime $y-b \ll b$, and the contribution from near the turning point reads as

$$
\delta L=2 \int_{z_{\star}} \frac{d z}{4 z^{\frac{5-a}{8}}(2 b)^{\frac{5+a}{8}}} \frac{1}{\sqrt{e^{\phi_{0}} q^{4} z^{|\beta|}(2 b)^{1+\beta}-1}} .
$$

Thus we conclude that

$$
\delta L \sim q^{-\frac{3+a}{2|\beta|}}
$$

which is negligible in large $q$ limit. Obviously, the intermediate region contributes only of order one to the integral. This shows that the separation has a maximum value for some $q$, which we denote by $L_{\max }$.

What really happens if the separation of the external quarks becomes larger than $L_{\text {max }}$ ? In this case, the strings follow the trajectory of the trivial solution, $\frac{d x}{d y}=0$. The strings from the boundary quarks and antiquarks are stretched straight toward the singularity without any change of $x$ coordinate. Very near the singularity corresponding to IR regime of the dual field theory, the strings are joined by changing $x$ coordinate. This situation is depicted in Figure 1 . One may worry about the part of the string very near the singularity. (This part describes the physics of the field lines in the extreme IR regime of the energy scale.). However one may see 
that the contribution to the energy of this part is zero at any rate. To see this, let us first note that the energy integral of the configuration is given by

$$
E_{s}=\sqrt{\lambda} \int \sqrt{A B d y^{2}+A C d x^{2}} .
$$

Since $d y=0$ for the part of joining two different straight strings,

$$
E_{\text {joint }}=\sqrt{\lambda} \int \sqrt{A C} d x=\left.\sqrt{\lambda} L \frac{(y-b)^{\frac{|\beta|}{2}}}{(y+b)^{-\frac{1+\beta}{2}}}\right|_{y=b}=0
$$

where in the last equality we have used the fact $\beta<0$. Thus the boundary condition at the singularity does not matter and the result of vanishing string energy has its own validity despite the singularity.

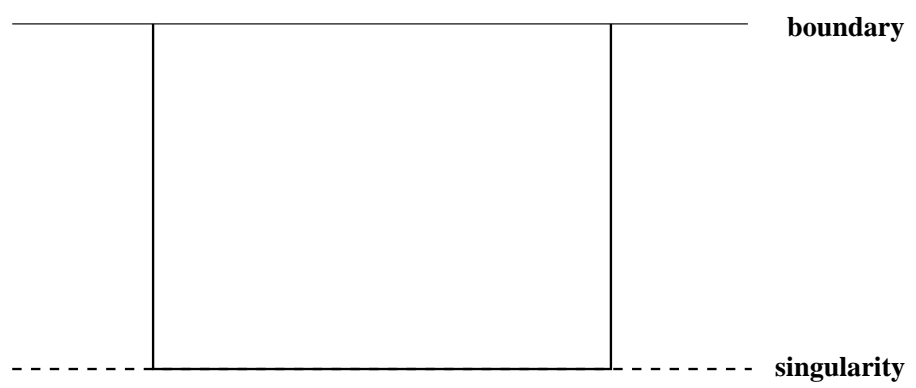

Figure 1: A string configuration touching the singularity represents the screening.

From the above discussion, the nature of the interaction is clear. The charges are not confined for $k / \mu>-12$. When the separation becomes larger than $L_{\max }$, the quark/anti-quark potential diminishes, representing the phenomenon of screening. Therefore we conclude that the regime of $k / \mu>-12$ corresponds to a screening phase.

\subsection{Confinement vs Screening of Heavy Quarks}

¿From the discussion above, one may expect that the system shows the phase transition as one varies the parameter $k / \mu$ by adjusting $k$ or $\mu$. At $k / \mu=-12$, the system undergoes the phase transition between a confinement phase and a screening phase for heavy quarks. The appearance of the tension of the electric-flux string in the confining phase may serve as an order parameter. At the critical point of $k / \mu=-12$ or $\beta \rightarrow 0$ limit, the electric-flux string tension takes a finite value of

$$
T_{Q C D}=5 \sqrt{\lambda \mu} e^{\frac{\phi_{0}}{2}}
$$


Namely the tension jumps to the finite value at the phase transition. This may be understood as follows. Due to the Gauss law, the total electric flux around charges should remain preserved irrespective of confinement or screening. Then, when quarks are confined by the transition, the electric flux lines form a linear tube and the finite tension simply comes from the existing energy of the field profile. Thus the tension should start with a finite value.

\section{4 "Doubly Screening Phase"}

In this subsection, let us consider the response of D-strings describing the interaction between magnetically charged objects. From the Lagrangian in (3.12), the dynamics of $(1,0)$ strings for a given $k$ is mapped into $(0,1)$ strings with $-k$. Thus, without further computation, one may see that magnetically charged quarks are confined when $k / \mu>12$ and screened otherwise.

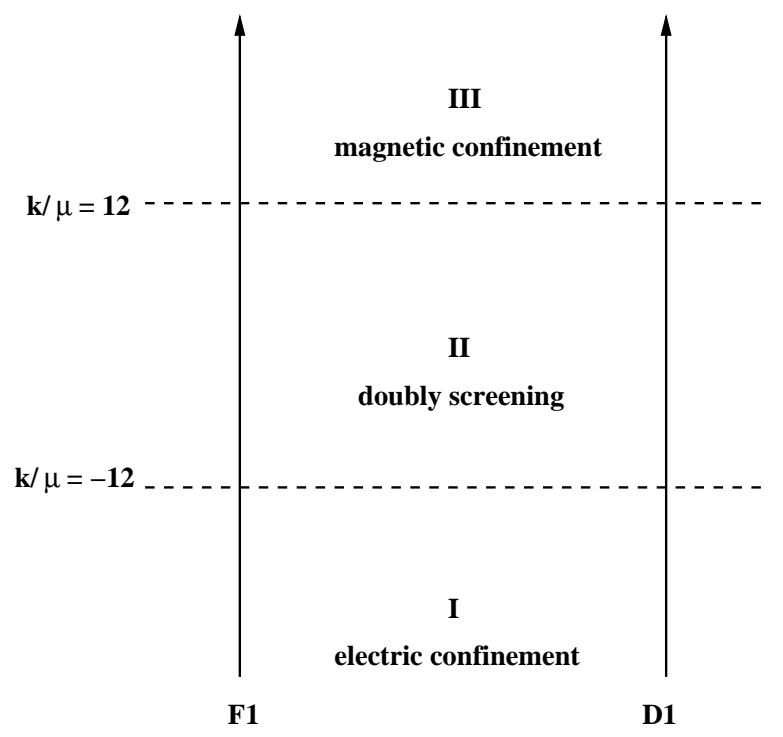

Figure 2: The full phase diagram.

The full phase structure is drawn in Figure 2. The region I with $k / \mu<-12$ describes the phase where the electrically charged quarks are confined. Then the magnetically charged objects should be screened, which is indeed the case as discussed above. The region III with $k / \mu>12$ corresponds to the phase where magnetic charges are confined while quarks are screened. This corresponds to the S-dual of the region I.

The region II with $-12<k / \mu<12$ describes the phase where both the quarks and magnetic charges are screened, which we call as 'doubly screening phase'. As far as we know, there were no such examples previously where both the electric and magnetic charges are screened. 
Presumably this phase structure is possible due to the S-duality symmetry of the underlying $\mathcal{N}=4$ SYM theory.

\section{Spontaneous Chiral Symmetry Breaking : D7 Probe Analysis}

\subsection{Generalities}

A few $N_{f}$ D7-branes parallel to a stack of large number $N$ of D3-branes introduces $N_{f}$ fundamental $\mathcal{N}=2$ hypermultiplets in the low energy gauge dynamics on D3-branes. For $N$ large enough, $N \gg N_{f}$, back reaction of the D7-branes to the near horizon limit of supergravity background may be irrelevant, and the low energy gauge dynamics on D3-branes with $\mathcal{N}=2$ fundamental hypermultiplets is supposed to be dual to $A d S_{5} \times S^{5}$ with probe D7-branes [5]. The open string dynamics on the probe D7-branes corresponds to the dynamics of $\mathcal{N}=2$ hypermultiplets in the "ambient" $\mathcal{N}=4 \mathrm{SYM}$ theory. This is because the gauge theory interpretation of the probe approximation is to take the quenched approximation neglecting effects of hypermultiplets to the dynamics of $\mathcal{N}=4 S U(N)$ SYM with large $N$. However, it should be noted that these probe $\mathcal{N}=2$ fundamental hypermultiplets experience full dynamics of $\mathcal{N}=4$ SYM theory.

In the D-brane picture in flat 10-dimensional space-time, let the D3-brane world-volume span along $\{0123\}$, and the D7-brane along $\{01234567\}$ directions. The distance between D3 and D7 in the transverse $\{89\}$ space gives rise to mass term in the Lagrangian for hypermultiplets. More specifically, asymptotic value of $w=\sqrt{x_{8}^{2}+x_{9}^{2}}$ for large $\rho^{2}=x_{4}^{2}+x_{5}^{2}+x_{6}^{2}+x_{7}^{2}$ of the D7 world-volume corresponds to the bare mass $m_{f}$ of the hypermultiplets ${ }^{2}$. For the maximally supersymmetric configuration, $w$ is constant on D7 (say, D7 lies at constant $\left(x_{8}, x_{9}\right)=\left(w_{0}, 0\right)$ while D3 is sitting at the origin). However, for non-supersymmetric states such as those we are considering, $w$ is generically a varying function of $\rho$.

In the supergravity picture, it is not difficult to identify the bare mass for the hypermultiplets in the framework of AdS/CFT correspondence. The maximally supersymmetric supergravity background in the near horizon limit is $A d S_{5} \times S^{5}$ with the string frame metric

$$
\begin{aligned}
d s^{2} & =\frac{1}{f(r)}\left(\sum_{\mu=0}^{3} d x_{\mu} d x^{\mu}\right)+f(r)\left(\sum_{i=4}^{9} d x^{i} d x^{i}\right) \\
& =\frac{r^{2}}{l^{2}}\left(\sum_{\mu=0}^{3} d x_{\mu} d x^{\mu}\right)+\frac{l^{2}}{r^{2}} d r^{2}+l^{2} d \Omega_{5}^{2}
\end{aligned}
$$

where $r^{2}=\sum_{i=4}^{9} x_{i}^{2}=\rho^{2}+w^{2}$ and $f(r)=\frac{4 \pi N g_{s}}{r^{2}}=\frac{l^{2}}{r^{2}}$ is the warping factor. The world-volume

${ }^{2}$ We use the notation $x_{i}=x^{i}$ for the spatial coordinates. 
profile of the probe D7-brane in this background is simply given by identifying the flat D-brane picture coordinate $\left\{x^{M}\right\}(M=0, \ldots, 9)$ with the coordinate $\left\{x^{M}\right\}$ in (4.36). For example, maximally supersymmetric D7 lying on the plane $\left(x_{8}, x_{9}\right)=\left(w_{0}, 0\right)$ fills the $A d S_{5}$ part of $\left\{x^{\mu}, r\right\}$ for $w_{0} \leq r<\infty$, in addition to wrapping $S^{3}$-cycle in the $S^{5}$. The wrapped $S^{3}$ is defined by $x_{4}^{2}+x_{5}^{2}+x_{6}^{2}+x_{7}^{2}=\rho^{2}=r^{2}-w_{0}^{2}$ and it vanishes at $r=w_{0}$, ensuring a smooth D7 world-volume in $A d S_{5} \times S^{5}$. Note that D7-brane is absent in energy scales below $r=w_{0}$; this is consistent with the field theory expectation that we shouldn't find any hypermultiplet below its mass scale $m_{f}=w_{0}$. Moreover, $w_{0}$ is a free parameter representing a family of the D7 profiles ; this gives us a freedom of changing the bare mass of hypermultiplets. Especially interesting limit would be the chiral symmetry limit ${ }^{3}$ of $m_{f}=w_{0}=0$.

For the non-supersymmetric backgrounds of our interest, it is possible to identify suitable coordinate $\left\{x^{M}\right\}$ that has a natural interpretation of flat coordinate in the D-brane picture. The string frame metric in this coordinate is ${ }^{4}$

$$
\begin{aligned}
d s^{2}=\left(\frac{r^{4}-1}{r^{4}+1}\right)^{\frac{k}{8 b}}\{ & -\left(r^{2}-r^{-2}\right)^{\frac{1+3 a}{2}}\left(r^{2}+r^{-2}\right)^{\frac{1-3 a}{2}} d x^{0} d x^{0}+\frac{1}{r^{2}}\left(\sum_{i=4}^{9} d x^{i} d x^{i}\right) \\
& \left.+\left(r^{2}-r^{-2}\right)^{\frac{1-a}{2}}\left(r^{2}+r^{-2}\right)^{\frac{1+a}{2}} d \vec{x} \cdot d \vec{x}\right\}
\end{aligned}
$$

where $r^{2}=\left(x_{4}^{2}+x_{5}^{2}+x_{6}^{2}+x_{7}^{2}\right)+\left(x_{8}^{2}+x_{9}^{2}\right) \equiv \rho^{2}+w^{2}$ as before. The above metric is obtained from (2.1) by combining $d y^{2}$ and $d \Omega_{5}^{2}$ with a change of variable $y=b \cosh (2 s)$ and $r^{2}=e^{s}$. The $R^{1,3}$ coordinate $\left\{x^{0}, \vec{x}\right\}$ has been also rescaled appropriately. The singularity is now positioned at $r^{2}=\rho^{2}+w^{2}=1$. The probe D7 world-volume covers $x^{0}, \ldots, x^{7}$ and its transverse position is given by $\left(x_{8}, x_{9}\right)=(w(\rho), 0)$ without loss of generality. Hence, it fills the (approximate) $A d S_{5}$ space over $w(0) \leq r<\infty$ (equivalently $0 \leq \rho<\infty$ ), and the wrapped $S^{3}$-cycle in $S^{5}$ shrinks to zero at $r=w(0)$. As we have explained in the previous paragraph, the field theory situation dual to this configuration is a quantum state of $\mathcal{N}=4 \mathrm{SYM}$ with $\mathcal{N}=2$ fundamental hypermultiplet in quenched approximation, whose bare mass is identified with $m_{f}=w(\infty)$. A family of profiles with varying $w(\infty)$ allows us to tune the bare mass, and in lucky cases, to get the chiral limit.

According to the AdS/CFT proposal, however, the bare mass is not the only information we can extract from $w(\rho)$. Viewing $w(\rho)$ as an effective scalar field in the $A d S_{5}$, its asymptotic value at $\rho \rightarrow \infty$ couples to some scalar operator in the field theory. We have actually identified this operator ; we have seen that $w(\infty)$ couples to the mass operator of the fundamental $\mathcal{N}=2$

\footnotetext{
${ }^{3}$ By chiral symmetry, we mean a chiral U(1)-symmetry which we discuss more in section (4.3).

${ }^{4}$ From now on, we set $\phi_{0}=0$ because it plays no special role except the trivial overall scaling.
} 
hypermultiplet,

$$
\begin{aligned}
\delta \mathcal{L}_{\mathrm{SYM}} & =w(\infty) \int d^{2} \theta \tilde{Q}_{f} Q_{f}-(\text { h.c. }) \\
& \sim w(\infty)\left(\tilde{q}_{L}^{f} q_{L}^{f}+\text { h.c. }\right)+(\text { bosonic })=w(\infty) \bar{q}_{D}^{f} q_{D}^{f}+(\text { bosonic })
\end{aligned}
$$

where we have introduced Dirac fermions,

$$
q_{D}^{f}=\left(\begin{array}{c}
q_{L}^{f} \\
i \sigma^{2}\left(\tilde{q}_{L}^{f}\right)^{*}
\end{array}\right)
$$

The fermion mass operator is of dimension 3, and the AdS/CFT dictionary tells us that its expectation value is encoded in the coefficient of sub-leading $\sim \frac{1}{\rho^{2}}$ behavior of $w(\rho)$ in $\rho \rightarrow \infty$. Note that the bosonic piece in the above has vanishing expectation value in the symmetric phase. As we have a freedom of choosing the bare mass $w(\infty)$, it is possible to see interesting dependence of bi-fermion mass operator condensate on the bare mass parameter. In the chiral limit, we may discuss about the occurrence of spontaneous chiral symmetry breaking.

\subsection{A Subtlety}

In this subsection, we show that the expectation value of the fermion mass operator for hypermultiplet is precisely given by the coefficient of sub-leading $\frac{1}{\rho^{2}}$ in $w(\rho)$ as $\rho \rightarrow \infty$. This is equivalent to a subtle question of choosing correct field variable, from whose asymptotic behavior we should read off the expectation value of the field theory operator. This is a relevant caveat to care about because $w(\rho)$ has a highly non-standard form of action functional in $A d S_{5}$ derived from the D7 DBI action. The relevant part of the D7-brane DBI action is

$$
S_{\mathrm{D} 7}=\tau_{7} \int d^{8} \xi e^{-\phi} \sqrt{-\operatorname{det}\left(\frac{\partial x^{M}}{\partial \xi^{\mu}} \frac{\partial x^{N}}{\partial \xi^{\nu}} G_{M N}^{(10)}\right)}
$$

where $G^{(10)}$ is the 10-dimensional metric of (4.37), and the dilaton profile is

$$
e^{-\phi}=\left(\frac{r^{4}+1}{r^{4}-1}\right)^{\frac{k}{4 b}}
$$

Choosing the gauge $\xi^{i}=x^{i}(i=0, \ldots, 7)$, and $\left(x^{8}, x^{9}\right)=(w(\rho), 0)$, we obtain the effective action for $w(\rho)$,

$$
S \sim \int d^{4} x \int_{0}^{\infty} d \rho \rho^{3} Z\left(\rho^{2}+w^{2}\right) \sqrt{1+\left(\frac{d w}{d \rho}\right)^{2}}
$$

where $Z(x)$ is a complicated function which goes to unity for large $x$;

$$
Z(x)=\left(1-\frac{1}{x^{4}}\right)\left(\frac{x^{2}-1}{x^{2}+1}\right)^{\frac{k}{4 b}} .
$$


Another fact that will be important for us later is $Z^{\prime}(x) \sim \frac{1}{x^{3}}$ for large $x$.

For a smooth D7 embedding, we need to impose the boundary condition, $\frac{d w}{d \rho}(0)=0$. In the asymptotic $\rho \rightarrow \infty$ region, $Z$ goes to unity and the solution of the equation of motion behaves as

$$
w(\rho) \sim m+\frac{C}{\rho^{2}}
$$

Naively, the $\rho$ integration in (4.42) diverges because for large $\rho$,

$$
\rho^{3} Z\left(\rho^{2}+w^{2}\right) \sqrt{1+\left(\frac{d w}{d \rho}\right)^{2}} \sim\left(\rho^{3}-\frac{k}{2 b} \frac{1}{\rho}\right)+\left(\frac{k}{2 b}\left(2 m^{2}+1\right)+2 C^{2}\right) \frac{1}{\rho^{3}}+\cdots
$$

and we need a suitable regularization procedure. However, what we are interested in will be variations of the value of (4.42) under changing $w(\infty)=m$, and for this purpose, it is enough to regularize (4.42) by subtracting the value of it at some fixed reference solution $w_{0}(\rho)$;

$$
S_{\mathrm{R}} \equiv \int d^{4} x \int_{0}^{\infty} d \rho \rho^{3}\left(Z\left(\rho^{2}+w^{2}\right) \sqrt{1+\left(\frac{d w}{d \rho}\right)^{2}}-Z\left(\rho^{2}+w_{0}^{2}\right) \sqrt{1+\left(\frac{d w_{0}}{d \rho}\right)^{2}}\right),
$$

which is now convergent due to (4.45). The standard AdS/CFT correspondence is then,

$$
\exp \left(i S_{\mathrm{R}}[m]\right)=\left\langle\exp \left(i \int d^{4} x m \bar{q}_{D}^{f} q_{D}^{f}\right)\right\rangle,
$$

where $S_{\mathrm{R}}[\mathrm{m}]$ is the above regularized action evaluated for the solution of the equation of motion with $w(\infty)=m$. Hence, we have

$$
\frac{\delta S_{\mathrm{R}}[m]}{\delta m}=\int d^{4} x\left\langle\bar{q}_{D}^{f} q_{D}^{f}\right\rangle
$$

In fact, it is not difficult to calculate the left-hand side of the above relation. Suppose that $w+\delta w$ is the solution of the equation of motion with $(w+\delta w)(\infty)=m+\delta m$ for infinitesimal $\delta m$. The variation of $S_{\mathrm{R}}[m]$ is

$$
\delta S_{\mathrm{R}}[m]=\int d^{4} x \int_{0}^{\infty} d \rho \rho^{3}\left(2 w Z^{\prime}\left(\rho^{2}+w^{2}\right) \sqrt{1+\left(\frac{d w}{d \rho}\right)^{2}} \delta w+Z\left(\rho^{2}+w^{2}\right) \frac{\frac{d w}{d \rho} \frac{d \delta w}{d \rho}}{\sqrt{1+\left(\frac{d w}{d \rho}\right)^{2}}}\right)
$$

The convergence of this expression may easily be seen from the property $Z^{\prime}(x) \sim \frac{1}{x^{3}}$, and we are allowed to perform integration by part for the second term. The resulting integrand which is proportional to $\delta w$ vanishes because $w(\rho)$ satisfies the equation of motion, and the surviving surface contribution at $\rho=\infty$ is

$$
\int d^{4} x \lim _{\rho \rightarrow \infty}\left(\rho^{3} Z\left(\rho^{2}+w^{2}\right) \frac{\frac{d w}{d \rho}}{\sqrt{1+\left(\frac{d w}{d \rho}\right)^{2}}} \delta w\right)=\int d^{4} x(-2 C \delta m)
$$


using $\rho^{3} \frac{d w}{d \rho} \sim-2 C$ and $\delta w \sim \delta m$ for large $\rho$. Comparing with (4.48), we thus have

$$
\left\langle\bar{q}_{D}^{f} q_{D}^{f}\right\rangle=-2 C
$$

\subsection{The Chiral Symmetry $U(1)_{c}$}

In this subsection, we discuss more about the chiral $\mathrm{U}(1)$-symmetry of the gauge theory we are considering. In fact, it is clear in the D-brane picture of D3-D7 system that there must be a global U(1)-symmetry which corresponds to the rotation of D7's position in the transverse $\left(x_{8}, x_{9}\right)$ plane. (Recall that the D3-branes are aligned along $\{0123\}$, while D7-branes are along $\{01234567\}$. Let us put $N$ D3-branes at $\left(x_{8}, x_{9}\right)=(0,0)$, and $N_{f}$ D7-branes at $\left(x_{8}, x_{9}\right)=$ $\left(w_{1}, w_{2}\right)$.) The distance between D3 and D7 introduces a mass term for $\mathcal{N}=2$ fundamental hypermultiplets,

$$
\left(w_{1}+i w_{2}\right) \int d^{2} \theta \tilde{Q}_{f} Q^{f}+\text { h.c. } \sim\left(w_{1}+i w_{2}\right) \bar{q}_{D}^{f} q_{D}^{f}+\text { (bosonic) . }
$$

The rotation $\left(w_{1}+i w_{2}\right) \rightarrow e^{2 i \alpha} \cdot\left(w_{1}+i w_{2}\right)$ of the D7-brane's position does not change anything on the D3-brane world-volume in the D-brane picture, and hence there should exist a compensating chiral rotation which is a global symmetry of the gauge theory. We should also expect the same chiral symmetry in the non-supersymmetric backgrounds of our interest, because the solutions preserve the $S O(6)$ symmetry of $S^{5}$, which includes $\left(x_{8}, x_{9}\right)$-plane rotation as a subgroup.

Looking at the superpotential term,

$$
\int d^{2} \theta \tilde{Q}_{f} Z Q^{f}+\int d^{2} \theta \operatorname{tr}(Z[X, Y])
$$

where $X, Y$ and $Z$ are adjoint chiral superfields in $\mathcal{N}=4 \mathrm{SYM}$ theory $\left(Z=X_{8}+i X_{9}\right)$, it is easy to realize that this symmetry is a $R$-symmetry. From the D-brane picture, we should assign charges 1 and 0 to $Z$ and $X, Y$ respectively. Then $d^{2} \theta$ has charge -1 (or $\theta_{\alpha}$ has charge $\frac{1}{2}$ ), and this forces us to take charge 0 for $\tilde{Q}_{f}$ and $Q_{f}$. The reason behind the $R$-symmetry is clear ; when we rotate D7-branes in the $\left(x^{8}, x^{9}\right)$ plane, the corresponding 10 dimensional type IIB Killing spinor of D3-D7 system with eight real components also rotates accordingly.

Note that $\tilde{q}_{f}$ and $q_{f}$ both have charge $-\frac{1}{2}$ under this $U(1)_{c}$. In terms of the Dirac spinor $q_{D}^{f}$, the charge is $\frac{1}{2} \gamma^{5}=\frac{1}{2}\left(\begin{array}{cc}-1 & 0 \\ 0 & 1\end{array}\right)$, and a non-vanishing expectation value of $\left\langle\bar{q}_{D}^{f} q_{D}^{f}\right\rangle$ will break this chiral symmetry spontaneously. Although $U(1)_{c}$ has a quantum anomaly which is proportional to $-N_{f} \times C_{2}(F)$, where $C_{2}(F)$ is the Casimir invariant of fundamental representation, it is negligible in the large $N$, t' Hooft limit 24, 25]. From the D-brane picture, it comes as a surprise that there is an anomaly for $U(1)_{c}$ in the effective field theory on D3, because this 
is a simple coordinate rotation in the $\left(x^{8}, x^{9}\right)$-plane. The resolution of the puzzle lies in the fact that D7-brane sources a non-trivial profile of RR-scalar $C_{0}$ around it, such that rotation in the $\left(x^{8}, x^{9}\right)$-plane induces a shift monodromy of $C_{0}$ field which is exactly proportional to the number of D7-branes, $N_{f}$ [13. The RR-scalar $C_{0}$, however, couples to the D3-branes by

$$
C_{0} \int \operatorname{tr}(F \wedge F)
$$

Therefore, the shift of the $\theta$ parameter due to the field theory anomaly of $U(1)_{c}$ rotation is precisely cancelled by the shift monodromy of the bulk field $C_{0}$, and the total anomaly is absent in the whole system. This may well be called an example of anomaly inflow ${ }^{5}$ (See also [26] for a related discussion).

\subsection{Separation Between S $\chi \mathrm{SB}$ and Confinement}

The equation of motion for $w(\rho)$ from the effective action (4.42) is somewhat complicated, and does not seem to have any analytic solutions. We have performed numerical analysis for solving the equation of motion, and have identified the asymptotic data, $m$ and $C$, for each solution. In the previous subsections, we have seen that $m$ corresponds to the bare mass of $\mathcal{N}=2$ fundamental hypermultiplets, while $C$ is directly proportional to the condensate of the bi-fermion mass operator for the hypermultiplets. Hence, a solution whose asymptotic behavior is characterized by $m=0$, but $C \neq 0$, signals that chiral symmetry is spontaneously broken in the gauge theory living on the boundary.

The effective action (4.42) for the probe D7-brane has a parameter

$$
\frac{k}{b}=2\left(\frac{k}{\mu}\right)\left(1+\frac{k^{2}}{6 \mu^{2}}\right)^{-\frac{1}{2}},
$$

representing a family of bulk type-IIB supergravity backgrounds, which in turn correspond to a family of homogeneous quantum states of $\mathcal{N}=4 \mathrm{SYM}$ theory with $\mathcal{N}=2$ hypermultiplets in AdS/CFT correspondence. In section 2, we analyzed "phases" of these states and observed that their phase structure is sensitive to the value of $k / \mu$ (equivalently, $k / b$ ). Specifically, for $k / \mu<-12,(k / b<-4.8)$, we have an electric confinement, while for $k / \mu>+12,(k / b>+4.8)$, magnetically charged objects are confined. An interesting phase seems to happen for $-12<$ $k / \mu<+12,(-4.8<k / b<+4.8)$, in which both electric charges and magnetic charges are screened.

The existence of spontaneous chiral symmetry breaking $(\mathrm{S} \chi \mathrm{SB})$, that is, whether there is a solution with $m=0$ but $C \neq 0$ in the bulk, also depends on the parameter $k / \mu$ (or $k / b$ ).

\footnotetext{
${ }^{5}$ We thank Jaemo Park for a discussion on this.
} 


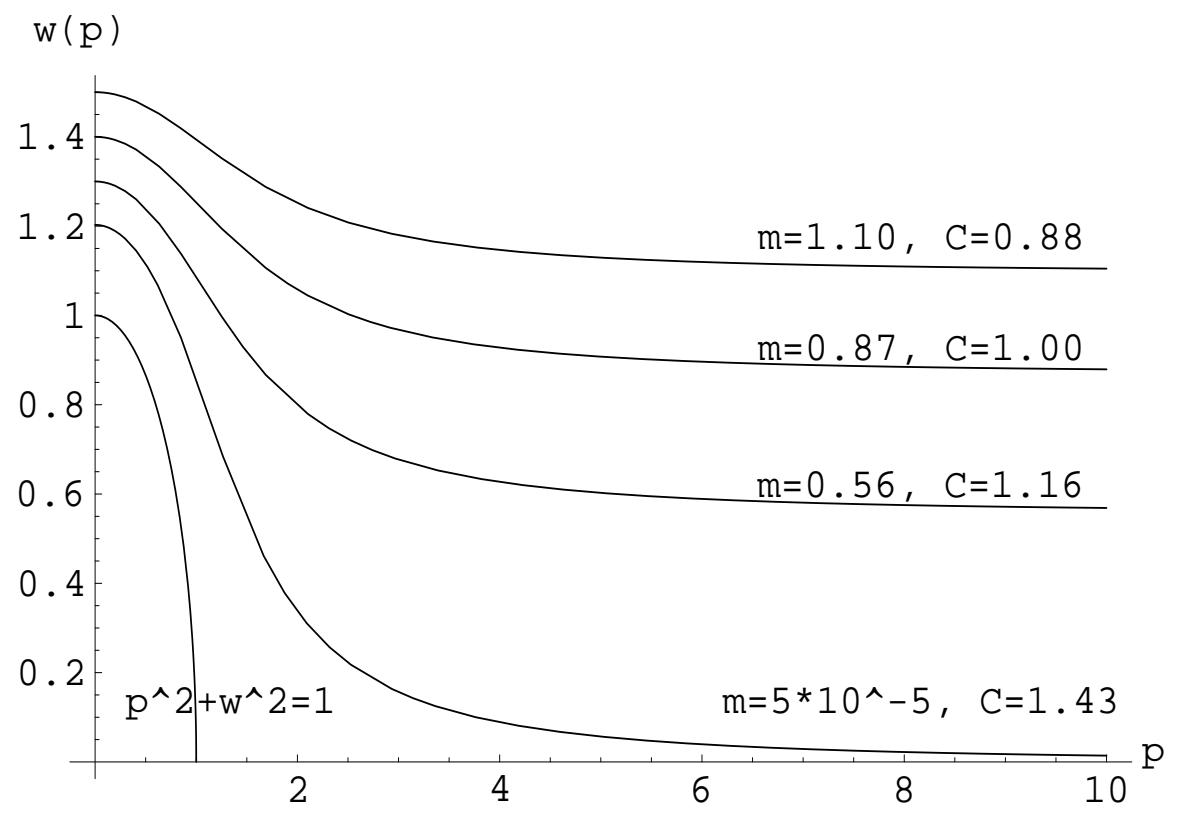

Figure 3: Numerical Solutions for $w(\rho)$ when $k / \mu=-7$ (or $k / b=-4.62$ ). It is clear that the solution with $m=0$, but $C \neq 0$, exists. The line $\rho^{2}+w^{2}=1$ is the position of the singularity.

Our numerical study shows that there is such a solution for $k / \mu<-2.97$ (or $k / b<-3.78$ ). As an exemplar case, Fig 3 is describing solutions with varying $w(\infty)=m$ when $k / \mu=-7$ (or $k / b=-4.62)$. It is evident from the figure that the value of $C$ does not vanish for the solution with $m=0$. What happens when $k / \mu>-2.97$ (or $k / b>-3.78$ ) is that solutions start to meet the singularity at $\rho^{2}+w^{2}=1$ as we lower the value of $m$. We thus cannot extract useful information for these cases.

The above analysis has a profound implication. For $-12<k / \mu<-2.97$ (or $-4.8<$ $k / b<-3.78)$, the corresponding quantum states of the gauge theory are in the screening phase, while massless fermions of fundamental representation form a non-vanishing bi-fermion condensation. This contradicts a prevailing lore that bi-fermion condensation would require a confining potential between two charges. On the basis of the AdS/CFT correspondence for probe D7-branes, we thus claim to have provided the first example of separation between spontaneous chiral symmetry breaking and confinement.

\section{Conclusion}

In this work, we have considered dilatonic deformations of AdS geometry that are dual to some quantum states of the $\mathcal{N}=4$ SYM theory with non-vanishing gluon condensation, $k$, as well as homogeneous energy density $\mu$. As varying the parameter $k / \mu$, we have identified the 
phases of these states by studying the interaction between quarks/anti-quarks, and also between magnetically charged objects. The regime $k / \mu<-12$ is electrically confining, where quarks are confined and magnetic charges are screened. The opposite regime of $k / \mu>12$ corresponds to the S-dual transformed phase, where magnetic charges are confined. For $-12<k / \mu<12$, interestingly both fundamental quarks as well as magnetic charges are screened, whose phase we call "doubly screening phase".

We then introduced the probe D7-branes and studied possible spontaneous chiral symmetry breaking. The $\mathcal{N}=2$ fundamental hypermultiplet arising from the D3-D7 strings possesses the classical chiral $U(1)_{c}$, which suffers from quantum anomaly. However we are working in the large $N$ limit of D3-branes and the effect of the anomaly may be ignored. By studying the D7 moduli dual to the fermion mass operator of the hypermultiplet, we have shown that there is a nonvanishing bifermion condensate in the zero-mass limit, leading to the spontaneous breaking of the chiral symmetry. We demonstrated that this happens even within the screening phase with no confinement.

It is our hope that the conclusions we have drawn from analyzing these states of $\mathcal{N}=4 \mathrm{SYM}$ theory reflect some truth of generic confining gauge theories. At least, it seems to suggest that spontaneous chiral symmetry breaking does not necessarily require confinement.

\section{Acknowledgement}

We would like to thank Kimyeong Lee, Jaemo Park and Soo-Jong Rey for helpful discussions. We also thank other participants of the "AdS/CFT and Quantum Chromodynamics" (Oct. 2830, 2004), Hanyang University, Korea, for inspiring discussions. D.B. is supported in part by KOSEF ABRL R14-2003-012-01002-0 and KOSEF R01-2003-000-10319-0. D.B. also likes to thank the warm hospitality of KIAS where part of this work was done. H.-U.Y. is partly supported by grant No. R01-2003-000-10391-0 from the Basic Research Program of the Korea Science \& Engineering Foundation.

\section{References}

[1] J. M. Maldacena, "The large N limit of superconformal field theories and supergravity," Adv. Theor. Math. Phys. 2, 231 (1998) [Int. J. Theor. Phys. 38, 1113 (1999)] arXiv:hep-th/9711200. 
[2] E. Witten, "Anti-de Sitter space and holography," Adv. Theor. Math. Phys. 2, 253 (1998) arXiv:hep-th/9802150.

[3] S. S. Gubser, I. R. Klebanov and A. M. Polyakov, "Gauge theory correlators from noncritical string theory," Phys. Lett. B 428, 105 (1998) arXiv:hep-th/9802109.

[4] D. Bak, M. Gutperle, S. Hirano and N. Ohta, "Dilatonic repulsons and confinement via the AdS/CFT correspondence," Phys. Rev. D 70, 086004 (2004) arXiv:hep-th/0403249.

[5] A. Karch and E. Katz, "Adding flavor to AdS/CFT," JHEP 0206, 043 (2002) arXiv:hep-th/0205236.

[6] A. Karch, E. Katz and N. Weiner, "Hadron masses and screening from AdS Wilson loops," Phys. Rev. Lett. 90, 091601 (2003) arXiv:hep-th/0211107.

[7] M. Kruczenski, D. Mateos, R. C. Myers and D. J. Winters, "Meson spectroscopy in AdS/CFT with flavour,” JHEP 0307, 049 (2003) arXiv:hep-th/0304032.

N. J. Evans and J. P. Shock, "Chiral dynamics from AdS space," Phys. Rev. D 70, 046002 (2004) arXiv:hep-th/0403279.

J. L. F. Barbon, C. Hoyos, D. Mateos and R. C. Myers, "The holographic life of the eta"," JHEP 0410, 029 (2004) arXiv:hep-th/0404260.

[8] J. Babington, J. Erdmenger, N. J. Evans, Z. Guralnik and I. Kirsch, "Chiral symmetry breaking and pions in non-supersymmetric gauge/gravity duals," Phys. Rev. D 69, 066007 (2004) arXiv:hep-th/0306018.

K. Ghoroku and M. Yahiro, "Chiral symmetry breaking driven by dilaton," Phys. Lett. B 604, 235 (2004) arXiv:hep-th/0408040.

[9] B. A. Burrington, J. T. Liu, L. A. Pando Zayas and D. Vaman, "Holographic duals of flavored N = 1 super Yang-Mills: Beyond the probe approximation," arXiv:hep-th/0406207.

[10] S. Hong, S. Yoon and M. J. Strassler, "Quarkonium from the fifth dimension," JHEP 0404, 046 (2004) arXiv:hep-th/0312071.

S. Hong, S. Yoon and M. J. Strassler, "On the couplings of vector mesons in AdS/QCD," arXiv:hep-th/0409118.

S. Hong, S. Yoon and M. J. Strassler, "Adjoint trapping: A new phenomenon at strong 't Hooft coupling," arXiv:hep-th/0410080.

M. Bando, T. Kugo, A. Sugamoto and S. Terunuma, "Pentaquark baryons in string theory," Prog. Theor. Phys. 112, 325 (2004) arXiv:hep-ph/0405259. 
[11] M. Rho, S. J. Sin and I. Zahed, "Elastic parton parton scattering from AdS/CFT," Phys. Lett. B 466, 199 (1999) arXiv:hep-th/9907126.

S. J. Sin and I. Zahed, "Holography of radiation and jet quenching," arXiv:hep-th/0407215.

[12] T. Sakai and J. Sonnenschein, "Probing flavored mesons of confining gauge theories by supergravity," JHEP 0309, 047 (2003) arXiv:hep-th/0305049.

S. Kuperstein, "Meson spectroscopy from holomorphic probes on the warped deformed conifold," arXiv:hep-th/0411097.

[13] P. Ouyang, "Holomorphic D7-branes and flavored $\mathrm{N}=1$ gauge theories," Nucl. Phys. B 699, 207 (2004) arXiv:hep-th/0311084.

[14] X. J. Wang and S. Hu, "Intersecting branes and adding flavors to the Maldacena-Nunez background," JHEP 0309, 017 (2003) arXiv:hep-th/0307218.

C. Nunez, A. Paredes and A. V. Ramallo, "Flavoring the gravity dual of N = 1 Yang-Mills with probes," JHEP 0312, 024 (2003) arXiv:hep-th/0311201.

[15] M. Kruczenski, D. Mateos, R. C. Myers and D. J. Winters, "Towards a holographic dual of large-N(c) QCD,” JHEP 0405, 041 (2004) arXiv:hep-th/0311270.

T. Sakai and S. Sugimoto, "Low energy hadron physics in holographic QCD," arXiv:hep-th/0412141.

[16] J. Erdmenger and I. Kirsch, "Mesons in gauge / gravity dual with large number of fundamental fields," JHEP 0412, 025 (2004) arXiv:hep-th/0408113.

[17] A. Kehagias and K. Sfetsos, "On running couplings in gauge theories from type-IIB supergravity," Phys. Lett. B 454, 270 (1999) arXiv:hep-th/9902125.

A. Kehagias and K. Sfetsos, "On asymptotic freedom and confinement from type-IIB supergravity," Phys. Lett. B 456, 22 (1999) arXiv:hep-th/9903109.

[18] S. Nojiri and S. D. Odintsov, "Running gauge coupling and quark anti-quark potential from dilatonic gravity," Phys. Lett. B 458, 226 (1999) arXiv:hep-th/9904036.

S. Nojiri and S. D. Odintsov, "Curvature dependence of running gauge coupling and confinement in AdS/CFT correspondence," Phys. Rev. D 61, 044014 (2000) arXiv:hep-th/9905200.

[19] D. Bak, M. Gutperle and S. Hirano, "A dilatonic deformation of AdS(5) and its field theory dual," JHEP 0305, 072 (2003) arXiv:hep-th/0304129. 
[20] M. A. Shifman, A. I. Vainshtein and V. I. Zakharov, "QCD And Resonance Physics. Sum Rules," Nucl. Phys. B 147, 385 (1979).

[21] S. J. Rey and J. T. Yee, "Macroscopic strings as heavy quarks in large N gauge theory and anti-de Sitter supergravity," Eur. Phys. J. C 22, 379 (2001) arXiv:hep-th/9803001.

[22] J. M. Maldacena, "Wilson loops in large N field theories," Phys. Rev. Lett. 80, 4859 (1998) arXiv:hep-th/9803002.

[23] S. S. Gubser, "Dilaton-driven confinement," arXiv:hep-th/9902155.

[24] E. Witten, "Current Algebra Theorems For The U(1) 'Goldstone Boson'," Nucl. Phys. B 156, 269 (1979).

[25] E. Witten, "Theta dependence in the large N limit of four-dimensional gauge theories," Phys. Rev. Lett. 81, 2862 (1998) arXiv:hep-th/9807109.

[26] A. Armoni, "Witten-Veneziano from Green-Schwarz," JHEP 0406, 019 (2004) arXiv:hep-th/0404248. 